\title{
Wirtschaftsinformatik-Forschung für die Praxis
}

\author{
Susanne Robra-Bissantz $\cdot$ Susanne Strahringer $(10)$
}

Eingegangen: 26. Januar 2020 / Angenommen: 18. Februar 2020 / Online publiziert: 27. Februar 2020

(C) Der/die Autor(en) 2020

Zusammenfassung Ganz im Sinne der Zeitschrift HMD - Praxis der Wirtschaftsinformatik existiert in Deutschland neben anderen Ausrichtungen auch eine explizit auf Praxisorientierung ausgerichtete Wirtschaftsinformatikforschung. Dieser Beitrag behandelt aus verschiedenen Blickwinkeln deren Entwicklungen und Strömungen. Im Mittelpunkt stehen Forschungsmethoden, die Forschung für die Praxis unterstützen. Dies sind insbesondere Methoden der Fallstudienforschung, der Aktionsforschung und der gestaltungsorientierten Forschung in verschiedenen Ausprägungen. Insbesondere diejenigen Methoden, die Forschung gemeinsam mit der Praxis anstreben, benötigen Rahmenbedingungen, die dies ermöglichen. Die gängigsten Kollaborationsformen, ihre Verknüpfung zu Forschungsmethoden, die von den jeweiligen Partnern zu erwartenden Nutzeffekte und Ergebnisse bilden den zweiten Schwerpunkt des Beitrages. Darüber hinaus identifizieren wir weitere Ansatzpunkte, die Forschern Wege aufzeigen, wie die Praxisrelevanz ihrer Forschung gesteigert werden kann. So gibt es methodische Bausteine, die vorhandene Forschungsmethoden um nicht invasive Komponenten ergänzen können, um die Relevanz der Forschungsergebnisse für die Praxis sicherzustellen. Zudem können Forscher auch an der besseren Konsumierbarkeit ihrer Ergebnisse arbeiten und sollten Mut fassen, die üblichen Routinen vergangener Forschung gelegentlich auch zu verlassen.

Schlüsselwörter Forschungsmethode · Design Science Research · Design Thinking · Aktionsforschung · Action Design Research · Fallstudienforschung

\footnotetext{
S. Robra-Bissantz

Institut für Wirtschaftsinformatik, Informationsmanagement, Technische Universität Braunschweig, Braunschweig, Deutschland

E-Mail: s.robra-bissantz@tu-bs.de

S. Strahringer $(\bowtie)$

Professur Wirtschaftsinformatik, insb. Informationssysteme in Industrie und Handel, Technische

Universität Dresden, Dresden, Deutschland

E-Mail: susanne.strahringer@tu-dresden.de
} 


\title{
"Wirtschaftsinformatik"-Research for Practice
}

\begin{abstract}
A considerable number of scholars in Germany conduct practice-oriented research that is in line with the aims and scope of the journal "HMD-Praxis der Wirtschaftsinformatik". This paper looks at this type of research from different perspectives and addresses research methods suitable for conducting research together with practice and suited for producing results applicable in practice. Against this backdrop, we discuss case study research, action research, and different flavors of design-oriented research. However, in order to conduct research together with practice in joint endeavors also organizational requirements have to be considered. We therefore present how collaboration between science and practice can be organized, how these forms of collaboration comply with research methods and what effects and outcomes are to be expected for each of the collaborating parties. Additionally, we identify other starting points that may help scholars in improving the relevance of their research to practice such as methodological building blocks that can be added to research methods non-invasively in order to ensure importance of research results to practitioners. Researchers can also work on making their research more accessible to practitioners. Ultimately, we also want to encourage researchers to sometimes deviate from the dominant and established ways of conducting research and to be open to new, risky, but perhaps more satisfying forms of research.
\end{abstract}

Keywords research method $\cdot$ design science research $\cdot$ design thinking $\cdot$ action research $\cdot$ action design research $\cdot$ case study research

\section{Praxisorientierte Forschung in der Wirtschaftsinformatik}

Die Wirtschaftsinformatik ist in ihrer Tradition ein Fach, in der die praktische Relevanz von Erkenntnissen schon seit Anbeginn im Fokus stand. Das heißt, sie entwickelte für Unternehmen Lösungen, wie Informationstechnik, Hard- und Software für verschiedene betriebliche Aufgaben möglichst gut eingesetzt werden kann. Dabei kannte die traditionelle, deutsche Wirtschaftsinformatik im Wesentlichen zwei Ausprägungen. Zum einen nutzte sie analytische Optimierungsmethoden, beispielsweise aus dem Bereich des Operations Research (OR), um IT-Systeme dazu zu befähigen für bestimmte Zielsetzungen, beispielsweise einer Reduzierung von Kosten, unter verschiedenen Restriktionen eine Lösung zu erarbeiten. Typisch hierfür ist die Optimierung in Produktion und Logistik. Die andere Ausprägung ist die des Software-Engineering, wobei ingenieurwissenschaftliche Ansätze, wie die Erhebung von Anforderungen und die fachliche und technische Beschreibung derer über Modellierung zu einem implementierten System führen. Sowohl das reine Anwenden von Optimierungsmethoden als auch das Entwickeln von Software mit großem Anwendungsbezug standen im Ruf, nicht ausreichend wissenschaftlich zu sein - eben keine wissenschaftliche Strenge (rigor) aufzuweisen (Gregor 2002). Das, was man als Wirtschaftsinformatik-Forschung hätte bezeichnen können, fand damit häufig im OR oder in der Informatik statt und folgte den in diesen Disziplinen üblichen Standards. 
Parallel wandelte sich mit zunehmendem Bewusstsein dafür, dass Technik nicht ohne den Menschen, ohne Organisationen und die Gesellschaft gedacht werden kann (Gregor und Jones 2007), die Bewertung, wann ein Informationssystem (aus Informationstechnik, betrieblicher Aufgabe und Mensch bestehend) als möglichst „gut“ gestaltet gilt. Statt allein Effizienz zu berücksichtigen, geht es heute auch um Kriterien der Effektivität, sodass der Technik-Einsatz zusätzlich Menschen zufriedenstellen oder zu Unternehmenszielen wie der Kundenbindung oder gesellschaftlichen Zielen wie der Nachhaltigkeit beitragen soll. Insbesondere dieser soziotechnische Fokus steht jedoch weder im OR noch in der Informatik im Vordergrund und prägt daher den spezifischen Zugang der Wirtschaftsinformatik bei der Problemlösung.

Anhänger von Forschung, die höchsten Ansprüchen hinsichtlich wissenschaftlicher Strenge (research rigor) genügen soll und dabei nicht per se auf die Lösung von Problemen der Praxis ausgerichtet sein muss, verfolgen in der Tradition der englischsprachigen Forschung des Information Systems (IS) Research vor allem eine empirische, verhaltensorientierte Forschung. Häufig stehen dabei Menschen oder Organisationen in Interaktion mit oder Wechselwirkung zu Informationstechnik im Zentrum einer eher sozialwissenschaftlichen Betrachtung. Ziel ist es, real $\mathrm{zu}$ beobachtende Phänomene zu erklären und entsprechende Theorien zu entwickeln (explanatory theories). Ein einfaches Beispiel, das über viele Jahre hinweg in der IS-Forschung genutzt wurde, ist das Technology-Acceptance-Modell, das aufzeigt, dass eine bessere Nutzbarkeit sowie eine höhere Nützlichkeit von Software zu einer höheren Akzeptanz derselben führen. Derartige Theorien können prinzipiell auch Vorhersagen treffen in der Form, dass eine Erhöhung der Nützlichkeit zu einer höheren Akzeptanz führt (predictive theories). Wenngleich diese Art von Forschung auch Anhaltspunkte geben kann, wie Systeme zu gestalten sind oder welche organisatorischen Verbesserungsmaßnahmen in Frage kommen, um zum Beispiel die Akzeptanz von Systemen zu erhöhen, stehen handlungsleitende Aussagen nicht im Mittelpunkt dieser Forschung. Dies hat Kritiker dazu verleitet, dieser Art von Forschung Praxisrelevanz abzusprechen und sie letztendlich für einen ,verfehlten“ Beitrag zu kritisieren.

Eine der Entwicklungen, die dazu geführt hat, dass Protagonisten beider Strömungen näher aneinander gerückt sind und Relevanz und Rigorosität (Robey und Markus 1998) nicht mehr als Widerspruch, sondern als vereinbar und unbedingt zu vereinbaren betrachtet werden, ist die seit ungefähr 20 Jahren in der Wirtschaftsinformatik und ihrer Schwesterdisziplin Information Systems geführte Diskussion um eine gestaltungsorientierte Wirtschaftsinformatikausrichtung, die im angelsächsischen Sprachraum unter der Bezeichnung ,Design Science Research“ (DSR) firmiert. Unter Verbindung wissenschaftlicher Strenge mit praktischer Relevanz werden Lösungsansätze entwickelt, die Gestaltungshinweise für Systeme und/oder ihren Einsatz geben und dabei die unternehmerische Aufgabe ebenso wie die beteiligten Menschen berücksichtigen (Iivari 2015). Dies ermöglicht die Verknüpfung von praktischer Nutzbarkeit mit wissenschaftlichem Erkenntnisgewinn. Dabei prägt sich zunehmend und über die Jahre hinweg ein gemeinsames Verständnis der Forschenden dazu aus, wie diese Art der Forschung noch besser wissenschaftlich fundiert und begründet durchgeführt werden kann, so dass daraus so genannte Design-Theorien entstehen, die auf eine Abstraktion der gefundenen Lösungen ausgerichtet sind 
(Gregor und Jones 2007), damit einem höheren Generalisierungsanspruch gerecht werden und der Extraktion übertragbaren Wissens dienen. In diesem Entwicklungsstrang ist insbesondere das vor knapp zehn Jahren von einer deutschsprachigen Professorengruppe veröffentlichte „Memorandum zur gestaltungsorientierten Wirtschaftsinformatik" (Österle et al. 2010) zu erwähnen, das später auch in englischer Sprache im European Journal of Information Systems (EJIS) (Österle et al. 2011) in den internationalen Diskurs eingebracht wurde. Trotz der Kritik an diesem Memorandum, z. B. hinsichtlich einer zu vereinfachten Charakterisierung der anglo-amerikanischen IS Community (Baskerville et al. 2011), reflektiert es eine Strömung, zu der sich die deutschsprachige Wirtschaftsinformatik in weiten Teilen bekennt.

Design Science Research kennt heute zahlreiche Varianten. Peffers et al. (2018) haben dies zum Anlass genommen, die diversen DSR-Schulen in ihrem EJIS-Editorial zu einem DSR-Schwerpunktheft in die wesentlichen fünf Genres zu klassifizieren, worunter die gestaltungsorientierte Wirtschaftsinformatik (Winter 2008; Österle et al. 2010) als eine Ausprägung betrachtet wird.

Es wäre jedoch verkürzend, Ansätze zu einer stark auf Praxisorientierung ausgerichteten Forschung in der Wirtschaftsinformatik nur im Umfeld von DSR zu suchen. DSR hat im Kontext von Praxisrelevanz eher Forschung für die Praxis im Fokus. Darüberhinausgehend adressieren die im Folgenden beschriebenen Strömungen Forschung in und gemeinsam mit der Praxis, die auch die Wirtschaftsinformatik erheblich beeinflusst haben.

Zum einen ist hier die klassische Fallstudienforschung zu nennen, bei der sich der Forscher typischerweise in die Praxis hineinbegibt, Daten sammelt, interviewt, ggf. beobachtet und dabei Phänomene in ihrem Kontext zu beschreiben, zu verstehen oder zu erklären versucht. Der Forschende arbeitet dabei zwar in der Praxis, greift aber nicht verändernd ein, sondern ist und bleibt Beobachter. Zum anderen sind die auf Veränderung der Praxis ausgerichteten Ansätze zu nennen, die auch auf einer engeren Kollaboration mit der Praxis und gemeinsamer Wissensproduktion basieren. Unter der Bezeichnung ,engaged scholarship“ ist ein insbesondere auf Van de Ven (2007) zurückgehender Ansatz zu verstehen, der u.a. auf Arbeiten von Boyer (1996) $\mathrm{zu}$,scholarship of engagement“ rekurriert. In diesen auch von anderen Forschern aufgegriffenen Ansätzen werden in der Verzahnung von Praxis- und Forschungsaktivitäten, der Interaktion von Praktikern mit Forschern und der Berücksichtigung verschiedener Stakeholder erhebliche Chancen gesehen. Van de Ven (2007) führt dies in erster Line auf den großen Unterschied zwischen Theorie- und Praxiswissen zurück, so dass Theoriewissen nicht einfach in Praxiswissen transferiert bzw. durch Abstraktion aus diesem gewonnen werden kann. Die Nicht-Berücksichtigung der Komplementarität der beiden Wissensarten ist eine der Ursachen eines Theorie-Praxis-Gaps, der durch gemeinsame Wissensproduktion (Ko-Produktion) überwunden werden kann:

I propose a method of engaged scholarship for expanding the capabilities of scholars to study complex problems and create the kind of knowledge that advances both science and practice. Engaged scholarship is defined as a participative form of research for obtaining the different perspectives of key stakeholders (researchers, users, clients, sponsors, and practitioners) in studying complex 
problems. By involving others and leveraging their different kinds of knowledge, engaged scholarship can produce knowledge that is more penetrating and insightful than when scholars and practitioners work on the problems alone. (Van de Ven 2007, S. 9)

Insgesamt unterscheidet Van de Ven vier verschiedene Formen des engaged scholarship, von denen zwei mit höheren Graden der Ko-Produktion einhergehen. Dies ist zum einen die Design- und Evaluations- und zum anderen die Aktionsforschung. Da Van de Ven alle Wissenschaften vor Augen hat, die auf eine gewisse berufliche Orientierung oder Profession ausgerichtet sind (d.h. neben der Betriebswirtschaftslehre, auch Fächer wie Medizin, Recht, Ingenieurwissenschaften), adressiert er mit Design- und Evaluationsforschung nicht vornehmlich Informationssysteme oder ISnahe Artefakte wie es in der IS-näheren DSR üblich ist, sondern jegliche Form von Vorgehensweisen, Regelwerken, Handlungsanweisungen, Programmen und Modellen, die in irgendeiner Form der Lösungen von praktischen Problemen in einer der adressierten Professionen dienen. Die Aktionsforschung zielt auf Veränderung durch Interventionen und löst dabei typischerweise organisationsspezifische Probleme, ohne aber die Wissensproduktion im Sinne einer Wissenschaftsdisziplin aus dem Auge zu verlieren (Mathiassen und Nielsen 2008).

Auch wenn die gestaltungsorientierte Forschung in der deutschsprachigen Wirtschaftsinformatik mehr Beachtung findet als das ,engaged scholarship“, so hat letzteres in Europa insbesondere im skandinavischen Raum große Beachtung gefunden (Mathiassen und Nielsen 2008) und u. a. von dort ausgehend auch Einfluss auf die deutschsprachige Wirtschaftsinformatik genommen.

Wie die genannten Entwicklungsströmungen zeigen, kann Forschung für die Praxis auf sehr unterschiedlichem Weg geschehen. Im folgenden Beitrag werden hierzu Methoden und Organisationsformen adressiert. Dabei liegt unser besonderer Fokus auf Ansätzen, die zugleich auch die Forschung mit der Praxis unterstützen. Wir gehen daher folgenden Leitfragen nach:

- Welche Forschungsmethoden eignen sich für Forschung für die und insbesondere auch mit der Praxis?

- Wie kann Forschung mit der Praxis organisiert werden? In welchen Formen/ Grundtypen ist sie anzutreffen?

- Gibt es neben Forschungsmethoden und Organisationsformen weitere Ansatzpunkte, um Praxisrelevanz von Forschung zu unterstützen?

Der restliche Beitrag ist entlang dieser drei Leitfragen strukturiert.

\section{Forschungsmethoden für die und mit der Praxis}

Die im folgenden ausgewählten Forschungsmethoden sind besonders für eine praxisrelevante Forschung unter Beteiligung der Praxis geeignet. Dabei liegt der Hauptfokus auf der Gruppe der gestaltungsorientierten Forschungsmethoden. Es werden aber auch andere methodische Ansätze vorgestellt, die zumindest ein Eindringen in den Praxiskontext und damit eine bessere Verankerung in der Praxis unterstützen. 


\subsection{Fallstudienforschung (case study research)}

Fallstudienforschung ist eine in der Betriebswirtschaftslehre, der Wirtschaftsinformatik und vielen Sozialwissenschaften angewandte Forschungsform, die die Erforschung komplexer gegenwärtiger Phänomene in ihrem Kontext ermöglicht, insbesondere dann, wenn Grenzen zwischen Phänomen und Kontext unscharf sind (Yin 2018, S. 5). Sie gehört zur Gruppe der qualitativ empirischen Methoden und geht (insbesondere bei einer positivistischen Grundhaltung) davon aus, dass der Forscher ein außenstehender Beobachter ist, der möglichst objektiv und unbeeinflusst die zu untersuchende Realität wahrnimmt, ebenso und mit ähnlichem Ergebnis wie dies auch ein anderer Forscher tun könnte. Auch wenn die objektive Wahrnehmbarkeit der Realität bei anderen wissenschaftstheoretische Grundposition in Frage gestellt wird, ist den meisten Varianten der Fallstudienforschung gemeinsam, dass der Forscher beobachtet und aufzeichnet und nicht aktiv am untersuchten Phänomen arbeitet oder gar Teil des untersuchten Systems ist. Ebenso wenig greift der Forscher steuernd ein, typischerweise partizipiert er also nicht in oder an dem, was er beobachtet. Der Forscher ist damit zwar in der Praxis, bleibt aber in seiner Rolle des Beobachters. Auch wenn Fallstudienforschung, weil sie in ihren Ergebnissen stärker in der Situation und dem Kontext verhaftet ist, in ihren Ergebnissen für ein Praxispublikum interessanter erscheint und diese ihre Problemsituationen besser reflektiert sehen, kann sie zwar ausgesprochen praxisrelevante Ergebnisse hervorbringen, diese jedoch nicht per se in Ko-Produktion mit der Praxis. Mit der Rolle des Forschers als Beobachter geht einher, dass Fallstudienforschung für das beobachtete Unternehmen nicht direkt mit einem unmittelbaren und klar erkennbaren Nutzen verbunden ist. Es ist vielmehr Aufgabe der Forschung klar zu identifizieren, welcher Nutzen sich für das Unternehmen ergeben kann. „What's in it for them“ muss in der Regel vom Forscher zusammen mit dem Unternehmen erarbeitet werden (Darke et al. 1998, S. 281) und geht meist in Richtung einer Situationsanalyse oder Positionsbestimmung, im Falle von multipler Fallstudienforschung, in der mehrere Fälle untersucht werden, auch in die Richtung eines Vergleiches, bisweilen sogar in die einer Art Benchmarking.

Fallstudienforschung wird eingesetzt, um Phänomene zu beschreiben, Theorien zu entwickeln oder gar, um sie zu testen. Gerade in der Theorieentwicklung in bislang weniger gut durch die Wissenschaft durchdrungenen Gebieten kommt ihr eine besondere Bedeutung zu. Die durch Fallstudienforschung entwickelten Theorien erreichen oft keinen sehr hohen Abstraktionsgrad, sie werden daher gelegentlich auch als „midrange theories“ bezeichnet. Gerade dies macht sie bisweilen aber für die Praxis greifbarer.

In die Gruppe der Fallstudienforschung fallen heute eine Bandbreite von qualitativ-empirischen Forschungsmethoden, die sich trotz zum Teil großer Ähnlichkeiten mehr oder weniger deutlich voneinander abgrenzen lassen. In einem zweiteiligen Editorial untersuchen Sarker et al. (Sarker et al. 2018a, b) Formen der ,ersten Generation" qualitativ-empirischer Forschung in der IS-Disziplin, denen sie verschiedene Varianten der Fallstudienforschung und ähnlicher Ansätze zuordnen: die explorative, die positivistische und die interpretative Fallstudie, die Grounded Theory Methode, die Ethnographie und die Hermeneutik. Auf Basis von vier Kriterien versuchen sie, 


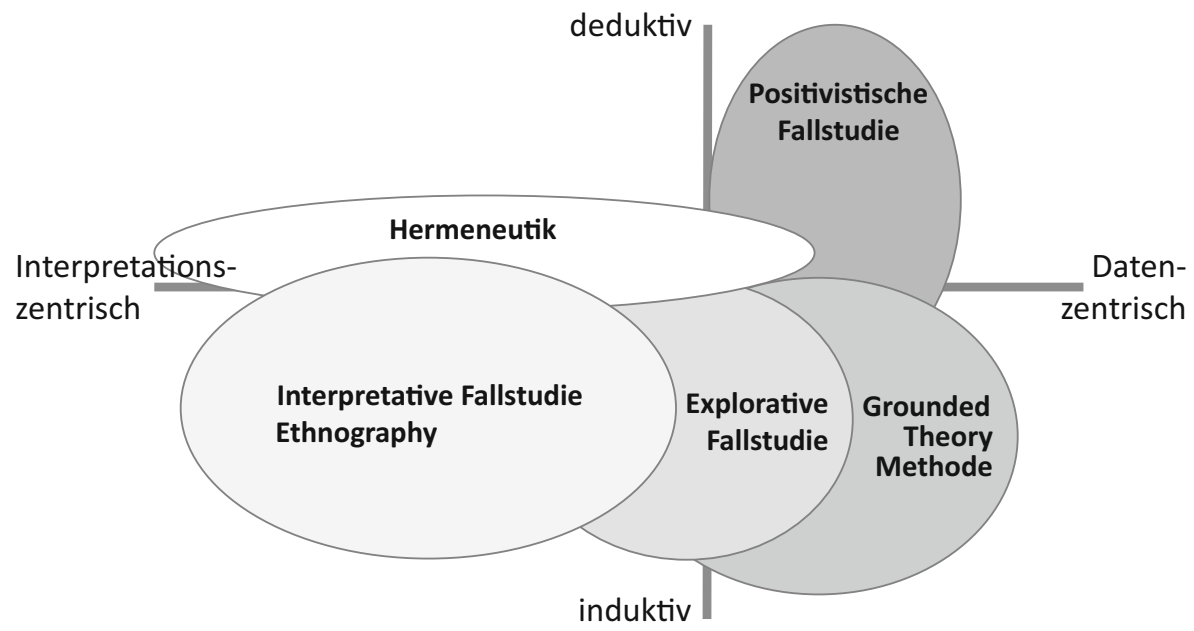

Abb. 1 Formen der Fallstudienforschung eingeordnet in eine Landkarte der ersten Generation qualitativer Forschungsmethoden. (Nach Sarker et al. 2018a, S. 762)

die heute in IS üblichen Genres abzugrenzen. Diese Kriterien sind (Sarker et al. 2018a, S. 756):

1. der Umgang des Forschenden mit Daten und sein Verständnis für Daten,

2. die Art und Rolle von Theorien in einer Studie,

3. die in der Studie gewählte Analyserichtung,

4. die Natur der aus der Studie folgenden Behauptungen oder Ergebnisse.

Verbindet man insbesondere die erste Dimension mit der grundlegenden Einteilung in induktive oder deduktive Forschung (dritte Dimension), so ergibt sich die in Abb. 1 dargestellte Abgrenzung.

Im Folgenden werden hiervon drei Formen betrachtet: die positivistische, die interpretative und die explorative Fallstudie (Sarker et al. 2018a, b).

In der positivistischen Fallstudie werden Daten als objektiv wahrnehmbar betrachtet und haben eine klare faktische Bedeutung. Sie können benutzt werden, um die Hypothesen einer Theorie zu falsifizieren, so dass oftmals ein deduktives, also theorietestendes Vorgehen zu beobachten ist. So prüfen beispielsweise Heier und Strahringer (2006) den Erklärungsbeitrag von Lewin/Scheins Change-Theorie bei der Analyse der Einführung des Wissensmanagementsystems ShareNet bei Siemens. In einer induktiven Variante diese Art der Fallstudienforschung geht es darum, Konstrukte und Beziehungen zwischen diesen im Sinne einer Theorieentwicklung zu identifizieren oder zu ergänzen.

In der interpretativen Fallstudie, die in der Klassifikation von Sarker et al. als große Gruppe mit vielen Spielarten betrachtet wird und auch die Ethnographie umfasst, werden Daten keine ihnen inhärente Bedeutung zugeschrieben, ihre Bedeutung ist immer subjektiv und Ergebnis von Interpretation und Zuschreibung. Der Forscher muss daher auch nicht außenstehender Beobachter sein, sondern kann wie in der Ethnographie üblich in die Welt, die er erforscht, eintauchen. Die Analyserichtung ist 
induktiv. Theorien dienen oftmals als eine Art Linse oder als ein Betrachtungsgerüst. So benutzen beispielsweise Mueller et al. (2019) in ihrer interpretativen Fallstudie die Theorie der sozialen Identität für die Interpretation der Perzeption von kritischen Erfolgsfaktoren in ERP-Programmen durch Stakeholder-Gruppen. Theorien können auch das Ergebnis interpretativer Studien sein, dann oftmals als so genannte „midrange theory“ oder als eine Art Framework. So identifizieren beispielsweise Sarker et al. (2012) Mechanismen der gemeinsamen Schaffung von Werten in B2BAllianzen bestehend aus ERP-Herstellern und deren Partnern. Diese Theorien können ideenreich sein oder auch pragmatische Zielsetzungen verfolgen. Sie werden typischerweise nicht als falsifizierbare Hypothesen formuliert. Das Ergebnis solcher Studien kann aber letztlich auch eine Re-Interpretation des untersuchten Phänomens darstellen.

Möglicherweise am schwierigsten abzugrenzen ist die explorative Fallstudie. So charakterisieren Sarker et al. (2018a, S. 762) dieses Genre als ,popular yet confusing“. Typischerweise ist sie induktiv, eher datenzentrisch, weniger interpretativ, aber hierin nicht klar positioniert. Die Analyse kann sich an diejenige anderer Formen anlehnen, erfolgt aber oft intuitiver. Ein theoretischer Zugang, wenn vorhanden, wird höchstens als grobe Leitlinie benutzt. Die Ergebnisse sind oftmals Frameworks, neue Einsichten oder Lessons Learned, die als provisorisch betrachtet werden und aufgrund ihrer betonten Vorläufigkeit eines weiteren Forschungsschrittes bedürfen. Als Beispiel lässt sich die Fallstudie von Kobus et al. (2018) anführen, in der Lessons Learned aus der Implementierung von Lean IT zur Ermöglichung von Digitalisierungsmaßnahmen abgeleitet werden.

Mit allen drei Fallstudienformen können praxisrelevante Ergebnisse erzeugt werden, jedoch führen insbesondere die induktiven Formen zu Ergebnissen, die für Praktiker näher an ihrer Lebenswelt wirken. Gerade explorative Fallstudien, die in plausiblen Frameworks und gut verständlichen Lessons Learned münden, sind für ein Praxispublikum am einfachsten zu verarbeiten und anzuwenden.

Eine wenig beachtete, besondere Variante der Fallstudienforschung ist die sog. partizipative Fallstudie, die Baskerville (1997) als eine dem Consulting ähnliche Aktivität positioniert, in der ein Forscher an der Verbesserung einer gegebenen Situation interessiert ist, sich nicht auf eine neutrale Sichtweise zurückzieht und dabei auch aktiv eingreift. Diese Form der Fallstudie ist allerdings von der Wissenschaftscommunity nicht weiterentwickelt oder in der Breite aufgegriffen worden, was letztlich auf die Problematik zurückzuführen ist, dass sie schwer als „Forschung“ zu verteidigen ist. Von Seiten der Praxis mag dies eine oftmals erwünschte Form der Zusammenarbeit sein, weil sie z.B. in Kontexten von Auftragsforschung die Umsetzung des Beauftragten ermöglicht. Dass Forscher aber immer Ergebnisoffenheit und den Beitrag auch für eine wissenschaftliche Community im Auge behalten müssen, und daher keine beauftragten Berater sind, weist auf die Risiken dieser Forschungsform hin.

Forschungsmethoden, die sich dieser Kritik deutlich verwehren und dabei dennoch einen Beitrag zur Lösung einer konkreten Problemsituation leisten, werden im folgenden Kapitel behandelt. 


\subsection{Aktionsforschung (action research)}

Aktionsforschung basiert auf der Idee, dass komplexe soziale Gefüge gut zu durchdringen sind, indem in ihnen Veränderungen implementiert und deren Effekte untersucht werden. Damit steht eine Intervention im Mittelpunkt, die konkrete Probleme adressiert und Veränderung herbeiführen soll. Die Extraktion von theoretischem Wissen wird jedoch nicht vernachlässigt. Die Aktionsforschung ist daher eine Form der Forschung, bei der der Forscher ähnlich wie bei der partizipativen Fallstudie aktiv eingreifen und problemlösend wirken kann, ohne sich der starken Kritik auszusetzen, nicht abgrenzbar von Beratung sein zu können: „it aims to solve current practical problems while expanding scientific knowledge" (Baskerville und Myers 2004, S. 329).

Aktionsforschung wird typischerweise in enger Kollaboration zwischen Forschern und Forschungssubjekten (Praktikern) sowie in Iterationen durchgeführt und fußt auf gemeinsamem Lernen im Kontext des sozialen Gefüges der beteiligten Praktiker (d.h. in der so genannten client-system infrastructure). Dabei werden zwei Abschnitte unterschieden: ein diagnostischer, in dem die soziale oder soziotechnische Situation gemeinsam analysiert und erste Theorien identifiziert bzw. entwickelt werden, und ein therapeutischer, in dem Veränderung implementiert und in ihren Wirkungen untersucht wird. Die Explikation einer Theorie vor der Intervention ist von besonderer Bedeutung, um die Intervention und ihre Zielsetzung zu begründen. Über das Ergebnis der Intervention in der Praxis wird die Theorie validiert und ggf. angepasst. Die Planung, Gestaltung und Umsetzung der Handlung sowie die Reflexion werden kollaborativ durchgeführt (Baskerville und Myers 2004).

Aktionsforschung bleibt trotz des Eingriffs in die Praxis und der potentiellen Erarbeitung konkreter Problemlösungen ein Forschungsprozess, der sich deutlich z. B. von der Beratung (siehe Tab. 1) unterscheidet.

Möglicherweise ist die Motivation des Praxispartners bei der Beteiligung an Aktionsforschung und der Beauftragung einer Beratung ähnlich, nämlich das Erreichen eines veränderten „,besseren“ Zustandes. Die Motivation der anderen Partei unterscheidet sich jedoch fundamental. Während der Berater nach dem eigenen geschäftlichen Erfolg strebt und bei seinem Kunden einen möglichst hohen Nutzen stiften möchte, ist er weder per se an der Verallgemeinerung des aus dem Projekt stammenden Wissens interessiert, noch daran es einer größeren Community zur Verfügung zu stellen. Vielmehr geht es in der Regel um die Produktion proprietären Wissens. Projektberichte etc. bleiben vertraulich und nur in positiven Fällen kann es auch zu Veröffentlichungen kommen. Aktionsforschung hingegen ist der Wissenschaftscommunity verpflichtet und muss dort einen Forschungsbeitrag zur Wissenschaft leisten, in der Regel über theoretisches Wissen, auch wenn dieses kontextverhaftet ist. Neben der Problemlösung für den Praxispartner steht die Dissemination des erzeugten Wissens im Vordergrund. Daraus ergibt sich auch die Verpflichtung in beiden Richtungen (Baskerville 1997).

In der Aktionsforschung wird die Analyse (Diagnose) gemeinsam als kollaborative Aufgabe durchgeführt, der Lösungsprozess ist eher experimentell, d. h. Forscher können keine spezifische Lösung oder gar Verbesserung versprechen und räumen ein, dass sie iterativ vorgehen und ggf. auch erfolglose Versuche haben werden. 
Tab. 1 Unterschiede zwischen Aktionsforschung und Beratung. (In Anlehnung an Baskerville 1997, S. 37)

\begin{tabular}{|c|c|c|}
\hline & Aktionsforschung & Beratung \\
\hline Motivation & $\begin{array}{l}\text { Beitrag zur Wissenschaft, theoretisches, } \\
\text { wenngleich situiertes Wissen, Publikation, } \\
\text { Interesse am Weg/Prozess }\end{array}$ & $\begin{array}{l}\text { Proprietäres Wissen, geschäftlicher Erfolg, } \\
\text { Interesse am Ergebnis }\end{array}$ \\
\hline $\begin{array}{l}\text { Verpflich- } \\
\text { tung }\end{array}$ & $\begin{array}{l}\text { Doppelt: sowohl Wissenschaftscommunity } \\
\text { als auch Praxispartner }\end{array}$ & Kundenunternehmen \\
\hline $\begin{array}{l}\text { Ansatz/ } \\
\text { Arbeits- } \\
\text { modus }\end{array}$ & $\begin{array}{l}\text { Kollaboration, Mitarbeit des Kunden in } \\
\text { der Rolle des „Praxisforschers“ ist essen- } \\
\text { tiell, iteratives kleinschrittiges Vorgehen } \\
\text { („,kleine“ Interventionen) }\end{array}$ & $\begin{array}{l}\text { Externe, unabhängige Sicht, z. T. Analyse } \\
\text { von außen, Kunden delegieren Aufgaben an } \\
\text { Berater, Kunden wählen aus vorgeschlage- } \\
\text { nen Lösungs-/Vorgehensalternativen }\end{array}$ \\
\hline $\begin{array}{l}\text { Empfeh- } \\
\text { lungs- } \\
\text { grund- } \\
\text { lage }\end{array}$ & Theorie, theoretische Frameworks & $\begin{array}{l}\text { Verlässliches Erfahrungswissen aus ähnli- } \\
\text { chen Situationen, vergleichbaren Problemla- } \\
\text { gen }\end{array}$ \\
\hline $\begin{array}{l}\text { Erkenntnis/ } \\
\text { Lösung }\end{array}$ & Durch Experimentieren & Durch kritische Analyse \\
\hline Erklärung & $\begin{array}{l}\text { Ideographisch und nur teilweise sowie } \\
\text { sehr schwer zu verallgemeinern }\end{array}$ & $\begin{array}{l}\text { Eher universelle Erklärungsmuster, die } \\
\text { von einem Fall auf den anderen transferiert } \\
\text { werden }\end{array}$ \\
\hline $\begin{array}{l}\text { Kunden- } \\
\text { nutzen }\end{array}$ & Durch situatives Lernen & $\begin{array}{l}\text { Durch Wissenstransfer in die Organisation } \\
\text { hinein }\end{array}$ \\
\hline
\end{tabular}

Berater werden hingegen oft als objektive Dritte und Außenstehende hinzugezogen, die die Situation kritisch analysieren und aufgrund ihrer professionellen Erfahrung und mit ihrem Erfahrungswissen aus vergleichbaren Kontexten mit Lösungsvorschlägen antreten. Typischerweise erwarten Kundenunternehmen von Beratern kein experimentelles Vorgehen, sondern zielsicheres und wollen auch einen Teil ihrer Aufgaben an diese delegieren. Bei der Erarbeitung von Lösungsansätzen und Interventionen stützen sich Aktionsforscher auf Theorien, die sie letztendlich validieren wollen. Auch wenn das Erfahrungswissen von Beratern eine ähnliche Qualität hat, ist es in der Regel nicht in Theorieform expliziert (Baskerville 1997).

Wir betonen diese Unterschiede, um auch in der Praxis ein Verständnis dafür zu schaffen, welche Unterschiede bei der Zusammenarbeit in Forschungsprozessen gegenüber der Nutzung von Beratung zu erwarten sind und wo Mitglieder der Wissenschaftscommunity gewöhnlich eine Grenze bei der Auftragsforschung ziehen.

\subsection{Gestaltungsorientierte Forschung (design science research)}

Design Science Research ist verwurzelt im Design Science - in der Wissenschaft der Gestaltung von Artefakten. Einfach formuliert sind Artefakte alles, was der Mensch künstlich gestaltet, um für sein Leben eine bessere Situation zu schaffen, als die Natur sie bietet (Simon 1996). Spätestens mit dem Bauhaus hat sich etabliert, dass das Gestalten (das Design) von Alltagsprodukten und Häusern mit Farben und Arbeitsabläufen eine Wissenschaft ist. Dabei spielten neue Technologien und die rationale, fundierte Begründung immer eine mehr oder weniger wichtige Rolle. Heute bezieht sich der Begriff des Artefaktes nicht allein auf Produkte, sondern 
ebenso auf Software, Dienstleistungen, Prozesse und gesamte Systeme aus Menschen, Dingen und Technologien sowie ihren Beziehungen untereinander (Redlich 2020). Ebenso wie das Design von Produkten sich auf grundlegende Theorien, wie beispielsweise die der Affordances oder verschiedene Farbkreise bezieht, nutzt und ergänzt DSR grundlegende Theorien, die Aufschluss darüber geben, wie das Artefakt möglichst gut gestaltet werden kann. Dazu gehören insbesondere empirisch fundierte psychologische oder sozialwissenschaftliche Theorien. Sie erklären für die Wirtschaftsinformatik beispielsweise, wann ein Mensch dazu bereit ist zu kollaborieren (explanatory theory) und entwickeln daraus normativ oder technologisch (im Sinne von Ziel-Mittel-Relationen) (prescriptive theory), wie eine IT-Plattform zu gestalten ist, um Kollaboration zu befördern.

Design Science Research beschränkt sich jedoch nicht darauf, gute Artefakte zu schaffen, sondern es entwickelt auch Ansätze zum Prozess, wie diese effektiv entstehen. In aller Munde ist hier das moderne Konzept des Design Thinking, das jenseits seiner Popularität Grundsätze formuliert, die auch im DSR der Wirtschaftsinformatik verankert werden können. Dazu gehört die Partizipation von Stakeholdern, wie sie bereits in der Aktionsforschung verhaftet ist. Im DSR entsteht daraus das Action Design Research, das von einem konkreten Unternehmensproblem ausgeht und dieses in Zusammenarbeit mit dem Unternehmen löst.

Häufig wird in der Wirtschaftsinformatik als Design Science Research auch bezeichnet, wenn das Ziel der Forschung nicht ist, ein Artefakt, wie eine neue digitale Plattform, zu gestalten, sondern ein Vorgehen, eine Methode oder grundlegende Prinzipien, wie diese zu gestalten ist. Dann steht im Vordergrund, den Gestaltungsoder Designprozess zu verbessern. Die entsprechende Forschung gibt Unternehmen Hinweise dazu, worauf sie achten müssen, wenn sie beispielsweise erwägen, in Zukunft mit Chat Bots im Kundenkontakt zu agieren. Aus dem Blickwinkel der Forschung entstehen damit Design Principles (Chandra et al. 2015) oder eine Design-Theorie, die aus konkreten Lösungen das Grundlegende abstrahieren, das für eine ganze Klasse von Lösungsansätzen gestaltungsleitend sein kann. Design-Theorien und Design-Prinzipien stellen daher auf andere Lösungen und Lösungsprozesse transferierbares Wissen dar. Im Vergleich zum klassischen Software Engineering, das mit Vorgehensmodellen und Patterns ähnliche Hilfestellung für eine ingenieurmäßige Softwarekonstruktion leistet, geht es aber hier nicht um Routinedesign, sondern um deutlich komplexere Probleme, nicht vornehmlich in technischer, sondern soziotechnischer Hinsicht, die es meist auch nicht optimal, sondern zufriedenstellend zu lösen gilt und deren gute Gestaltung eine Herausforderung darstellt. Im Gegensatz zu Prozessen und Hilfsmitteln, die man dem klassischen Software Engineering zurechnen könnte, geht es hier auch darum, unerwartete Lösungen zu entdecken (Hevner et al. 2004; Maedche et al. 2019; Gregor und Jones 2007).

Relativ neu ist die Erkenntnis, dass der typische und gute Designer über einzigartige Fähigkeiten im Gestaltungsprozess verfügt (Gregor 2002). Diese Fähigkeiten können unter Designerly Thinking zusammengefasst werden (Redlich 2020). In der wissenschaftlichen Befassung mit Design Research entstehen damit für Unternehmen Ansätze dafür, worauf sie in Gestaltungsprozessen von neuen Artefakten achten müssen und welche Methoden, beispielsweise in der Analyse des Problemraums (Maedche et al. 2019) dafür zur Verfügung stehen. 
Zusammengefasst werden damit im Folgenden neben der Grundidee des Design Science Research drei weitere Ausprägungen unterschieden und in Bezug darauf erklärt, wie sie mit der und für die Praxis zu Erkenntnissen führen.

1. Das Design Science Research (DSR), das von typischen, beobachteten Problemen der Praxis ausgeht und diese mit wissenschaftlichen Grundlagen auf hoher Abstraktionsebene löst.

2. Das Action Design Research (ADR), das von dem Problem eines einzelnen Unternehmens ausgeht und dieses partizipativ mit dem Unternehmen löst.

3. Die Gestaltung von Design-Theorien (DT), die den Abstraktionsgrad von DSRErgebnissen weiter verstärkt und damit die Übertragbarkeit des Design-Wissens fördert.

4. Das Designerly Thinking, das weitere Aspekte des Design-Prozesses identifiziert, die Unternehmen berücksichtigen sollten.

\subsubsection{Grundkonzept}

Das ursprünglichen Konzept des Design Science Research (DSR) (Hevner et al. 2004; Hevner 2007) strebt ganz grundsätzlich danach aufzuzeigen, dass das Gestalten (Design) von Lösungen mit Informationstechnologie, z. B. von Software, ganz eng mit der verhaltens- und sozialwissenschaftlichen Forschung zusammenhängt. Denn es liegt in der Natur eines modernen Unternehmens, dass es mit innovativer IT seine Prozesse und Strategien, an welchen Menschen beteiligt sind oder die auf Menschen zielen, verbessern will.

Die Forschung der Wirtschaftsinformatik entwickelt im DSR IT-Artefakte für Problemklassen, evaluiert sie und entwickelt sie weiter. Häufig sind dies so genannte Instanziierungen: meistens keine kompletten lauffähigen Systeme, sondern Prototypen oder Ausschnitte aus Gesamtsystemen. Dazu gehören aber auch andere Konstrukte, Modelle oder Methoden, die der Implementierung der Software vorgelagert sind. Hevner und Gregor (2013) sprechen mit Blick auf den zunehmenden Abstraktions- und Reifegrad bei Instanziierungen von Level-1-, bei Konstrukten, Modellen und Methoden von Level-2-Artefakten.

Diese IT-Artefakte sind aus vier Perspektiven wissenschaftlich untermauert und genügen daher den Anforderungen der Wissenschaftlichkeit. Zum einen basiert die Entwicklung der IT-Artefakte auf so genannten Kernel-Theorien (fachspezifische Theorien) aus den Verhaltens- und Sozialwissenschaften sowie auf bereits bestehenden Erkenntnissen der IS-Forschung. In der Forschung zur Groupware-Systemen ist beispielsweise aus den Theorien der Evaluation Apprehension bekannt, dass Anonymität unter den Gruppenmitgliedern dazu führt, dass sie eher bereit sind, neue Beiträge zu leisten. Eine prototypische Software wird daher Anonymität ermöglichen. Zweitens umfasst die Gestaltung der Artefakte deren Evaluation, die ebenfalls auf wissenschaftlichen Methoden basiert. Beispielsweise wird der genannte Prototyp mithilfe von verhaltenswissenschaftlichen Experimenten darauf getestet, ob er zum gewünschten Ziel führt. Sowohl die verschiedenen theoretischen Grundlagen als auch die Evaluationsmethoden finden sich in einer Wissensbasis, die sozusagen den Werkzeugkasten des Design-Science-Forschers darstellt. Die Ergebnisse der Eva- 
luation bestätigen oder modifizieren ggf. auch die zugrundeliegende Theorie. Und neue Erkenntnisse werden der Wissensbasis zugeführt. Dieser Kreislauf beschreibt den so genannten Rigor Cycle, der zusammen mit dem Design Cycle (mehrfaches Durchlaufen von Gestalten und Evaluieren von IT-Artefakten) den dritten Baustein gesicherter wissenschaftlicher Erkenntnis beiträgt. Der Forschende lernt aus allen Erkenntnissen, die er während seiner Designzyklen erwirbt, bleibt aber gleichzeitig über den weiteren Abgleich mit grundlegenden Theorien offen für neue Gestaltungsideen. Mehrere Design Cycles, die auch von unterschiedlichen Forschern durchlaufen werden, sichern (als vierte Perspektive), dass das Problem - wie es in der Problemstellung gefordert ist - einen gewissen Abstraktionsgrad hat und die Lösung generalisiert werden kann. Hier zeigen sich in neueren Diskussionen zwei Entwicklungen: zum einen wird zunehmend darauf verwiesen, dass die Wissensbasis zu den Kernel-Theorien auch Wissen in Form von Solution Design Entities (also Wissen zu erarbeiteten IT-Artefakten) sowie in Form von Solution Design Theories umfasst (Hevner et al. 2018). Letztere unterstützen als Design-Theorien DesignProzesse (vgl. Abschn. 2.3.3). Zum anderen entstehen Design-Theorien unter bestimmten Voraussetzungen als so genanntes Level-3-Artefakt aus den einzelnen ITArtefakten der beiden unteren Levels, indem beispielsweise mehrere Instanziierungen Erkenntnisse generalisieren und evaluieren (Gregor und Hevner 2013).

Trotz der wissenschaftlichen Vorgehensweise bleibt als eine Kernanforderung des Design Science Research, für die Praxis relevante Ergebnisse zu schaffen. Das Design von IT-Artefakten beruht daher immer auf Anforderungen, Bedarfen oder auch Problemen der Praxis - im Zusammenspiel von Mensch, Organisation, betrieblicher Aufgabe und Technologie. Obwohl DSR zulässt, dass der Forscher seine Problemstellung unter entsprechender Prüfung auf Praxisrelevanz selber entwickelt, können auch Unternehmen die Forschung mit einem eigenen Problem initiieren oder mit einem Ziel, das sie sich von einer neuen IT-Lösung erhoffen.

Spätestens in der Evaluation von IT-Artefakten sieht der so genannte Relevance Cycle des Design Science Research auch vor, die Prototypen im praktischen Umfeld bei Unternehmen zu testen. Für die Wissenschaft der Wirtschaftsinformatik ist eine derartige, praktische Evaluation Bestandteil der Forschung und sie ist daher, beispielsweise im Rahmen von Dissertationen oder für gemeinsame Projekte (z. B. BMBF-Projekte) häufig auf der Suche nach Kooperationspartnern aus der Praxis.

Auch für Unternehmen, die nicht direkt am Forschungsprozess beteiligt sind, entstehen als Ergebnisse des DSR IT-Artefakte, die als Blaupausen zumindest für einzelne Aspekte in ihrer weiteren Digitalisierung, für neue Software oder für die Weiterentwicklung ihrer IT-Infrastruktur dienen können. Denn grundsätzlich sieht das DSR vor, ihre Erkenntnisse aus IT-Artefakten zumindest zu Meta-Artefakten oder Design-Prinzipien weiterzuentwickeln (vgl. Abschn. 2.3.3). Die Veröffentlichung von Ergebnissen des DSR ist daher nicht nur in Formaten für die gemeinsame Weiterentwicklung der wissenschaftlichen Wissensbasis wichtig, sondern auch in praxisorientierten Zeitschriften wie der HMD - Praxis der Wirtschaftsinformatik. 


\subsubsection{Action Design Research}

Ebenso wie das Design Science Research wird auch das Action Design Research (ADR) noch diskutiert und mit unterschiedlichen Schwerpunkten vorgestellt. Vom Prinzip her verbindet es das Ziel der Gestaltung eines technologie-getriebenen Artefakts aus dem Design Science Research mit der Ausrichtung der Aktionsforschung auf die partizipative Entwicklung von Lösungen mit ihren Stakeholdern (vgl. Abschn. 2.2). Im Gegensatz zur reinen Aktionsforschung ist der Unterschied darin zu sehen, dass die Intervention auch in der Einführung einer IT-Lösung zu sehen ist und eines Gestaltungsprozesses bedarf, der damit über z. B. rein organisationale Interventionen hinausgeht. Im Vergleich zum herkömmlichen DSR weist ADR zusammenfassend wesentliche Besonderheiten in den drei Bereichen Problemstellung, angestrebtes Ergebnis sowie Gestaltungsprozess auf (Sein et al. 2011; Iivari 2015).

In der Problemstellung ergeben sich aus der Zusammenarbeit zwischen dem Unternehmen und den Forschenden besondere Chancen aus dem in der Wirtschaftsinformatik komplexen Zusammenspiel von Technologie und Organisation. Eine Chance für den Forscher entsteht daraus, Wissen aus dem besonderen, beobachtbaren Problem zu ziehen (Practice inspired Research). Dabei besteht eine Herausforderung darin, das Problem zu erklären, zu strukturieren und, für eine spätere Generalisierung, einer Problemklasse zuzuführen. Eine andere Chance besteht darin, bestehende Theorien im organisatorischen Umfeld über IT-Artefakte sozial erkennbar zu machen und damit aus ihnen erlebbare Lösungen zu schaffen (Theory ingrained Artifact). Action Design Research verbindet damit Theorie und Praxis sowie Denken und Tun.

Das Ergebnis des Action Design Research sind so genannte Ensemble-Artefakte, die explizit die Menschen aus der Organisation mit einbeziehen. Im klassischen DSR ist dies nicht zwingend. Damit bezieht die Erkenntnis auch ein, wie Menschen beispielsweise ihr Mindset oder ihre eigenen Prozesse im Zusammenspiel mit der IT verändern. Es ist eine Besonderheit des Action Design Research, dass der Forschende zwar IT-Artefakte aus Theorien entwickelt, diese sich aber in mehreren Zyklen im Kontext des Unternehmens und im Zusammenspiel mit den beteiligten Personen in besonderer Weise weiterentwickeln. Das Action Design Research schließt dabei gewöhnlich mit einem IT-Artefakt, das dem Unternehmen, so wie es im Forschungsprozess in seinem Einsatz weiterentwickelt wurde, fertig zur Verfügung steht.

Der Prozess des Action Design Research, beschrieben als „Building“, „Intervention“, „Evaluation“, hebt gezielt die Unterteilung des Design Science Research in die voneinander abgetrennten Phasen der Gestaltung und der Evaluation auf. Gemeinsam und in Diskussion z. B. der Ergebnisse vorheriger Zyklen wird das Ensemble-Artefakt gestaltet, in das organisatorische Umfeld getragen (Intervention), dort geformt und angepasst sowie fortlaufend evaluiert. Action Design Research begründet sich in einer aufeinander aufbauenden Zusammenarbeit und einer möglichst langfristig ausgelegten Beziehung zwischen Forscher und Unternehmen. Für die Wirtschaftsinformatik ist das Prinzip des so genannten ,Reciprocal Shaping“ im Design des Ensemble-Artefakts besonders interessant. Da die Technologie, mit jeweils neuen IT-Artefakten, und die Organisation, die diese in ihrem Umfeld nutzt, untrennbar sind, ist im Action Design Research gefordert, dass die jeweiligen Gestaltungsvor- 
schläge gezielt aufeinander eingehen und immer die Ergebnisse der Intervention widerspiegeln.

Für Unternehmen und Forscher stellt die Beteiligung am Action Design Research eine besondere Chance dar, denn diese Forschung verbindet die Entwicklung eines fertigen IT-Artefakts mit seiner Einbettung in das Unternehmen. Zudem macht Action Design Research Forschung und Theorien erlebbar - und gibt dafür Einsichten in komplexe Praxisprobleme zurück. Die Kooperation muss jedoch bereits mit der gemeinsamen Problemidentifikation starten, langfristig ausgelegt und im Unternehmen auch bezüglich des Zeitaufwands der Team-Mitglieder verankert sein.

\subsubsection{Gestaltung von Design-Theorien}

Design Science Research und Action Design Research nutzen die Gestaltung, das Design, als Forschungsmethode (Research) für Informationssysteme. Beschäftigt man sich mit dem Artefaktdesign an sich und hebt dieses auf eine höhere Abstraktionsstufe als dies beim DSR oder ADR notwendig wäre, entstehen, für einige Wissenschaftler als wesentliches Ergebnis gestaltungsorientierter Forschung, Design-Theorien (DT, so genannte Level-3-Artefakte), die Mittel-Zweck-Beziehungen aufzeigen und sowohl normative als auch präskriptive Aussagen beinhalten können (Fischer et al. 2010). Design-Theorien umfassen als „Theories of Design and Action" (Gregor 2002) die Gestaltungsgrundsätze für das Artefakt sowie Methoden und Tools für den Gestaltungsprozess.

Damit unterstützen DT den Designer, wenn er für ein Problem, für ein Ziel oder für bestimmte Anforderungen ein IT-Artefakt gestalten will. Mithilfe von DesignPrinzipien geben sie beispielsweise auch Unternehmen eine Art Rezept für die Gestaltung des Design-Prozesses und des IT-Artefakts vor. Diese müssen natürlich auf spezielle, auch zukünftige Situationen anpassbar, über die für ihre Entwicklung herangezogenen Theorien begründet und getestet sein. Ein Beispiel ist eine Canvas, die aufzeigt, welche Elemente in der Implementierung berücksichtigt werden müssen, oder eine Methode, wie man Anforderungen der Stakeholder ermittelt.

Design-Theorien der Wirtschaftsinformatik und des Information Systems Research können heute, aufgrund ihrer breit anerkannten „Anatomie“, der Konservierung und der Kommunikation von Design-Wissen dienen (Gregor und Jones 2007).

Wie Design-Theorien für IT-Artefakte entstehen, wird seit fast 30 Jahren (Walls et al. 1992) unter Rückgriff auf das Produktdesign (The Design of the Artificial, Simon 1969, 1996) diskutiert.

In der Tradition des Produktdesigners steht, auf gute, bereits existierende Designs zurückzugreifen - nicht aus Faulheit, sondern weil das bestehende Design bereits Wissen zu einem guten Design beinhaltet. Auch die Entstehung von Theorien aus dem Design Science Research nutzt jeweils Wissen aus der Gestaltung vorheriger IT-Artefakte. Dieses ist zusätzlich immer durch Kernel-Theorien fundiert und gelangt damit zu theoretisch fundierten Morphologien für Artefakte. Unternehmen können an einer solchen Theorieentwicklung durchaus teilhaben, indem sie für ihr Problem eine entstehende Design-Theorie anwenden (lassen) und damit zum Test ihrer allgemeinen Gültigkeit beitragen. Wichtig für die Wissenschaft ist, das Problem möglichst abstrakt zu formulieren, um wiederholte Forschungen am gleichen The- 
ma zu vermeiden bzw. um in dieser Form bestehende Grundlagen für weitergehende Forschung zu nutzen. Die Forschung zur Externalisierung von Expertenwissen beispielsweise, müsste dann nicht für das Wissensmanagement, Expertensysteme und betriebliche Nachfolgeregelungen einzeln erfolgen, sondern sie könnte immer auf bereits bestehendes Design-Wissen zurückgreifen.

Zunehmend entstehen Beiträge, die zu Design-Theorien im Prozess des Designens führen können. Sie zeigen beispielsweise auf, dass Design-Prinzipien sich, in der Tradition der Affordances des Produktdesigns, auf Aktionen der Nutzer des IT-Artefakts beziehen müssen, auf die einzelnen Komponenten, aus denen dieses bestehen soll, und auf besondere Randbedingungen des Umfelds (Chandra et al. 2015). Andere Beiträge erarbeiten, wie man das Problem, das mit dem IT-Artefakt gelöst werden soll, gut beschreiben oder gemeinsam erarbeiten kann: aus dem Bedarf, dem Ziel, den Anforderungen - unter Berücksichtigung der Stakeholder. Dies beruht auf dem grundsätzlichen Prinzip des Designers, Lösungs- und Problemraum gleichzeitig zu betrachten (Maedche et al. 2019).

Design-Prinzipien, die sich nicht nur auf IT-Artefakte beziehen, sondern auf den Prozess ihrer Gestaltung, sind für Unternehmen ganz wesentliche Beiträge der Forschung, wenn sich ihr Umfeld in der digitalen Transformation derart wandelt, dass es nicht mehr ausreicht, bekannte Probleme mit neuen Technologien besser zu lösen. Denn insbesondere Wissen zu Gestaltungsprozessen kann helfen, wenn Unternehmen ihr Informationssystem mit völlig veränderten Herausforderungen konfrontiert sehen, die aus der Digitalisierung resultieren und mit dieser gelöst werden müssen.

\subsubsection{Designerly Thinking}

Unter dem Stichwort des Designerly Thinking (Johansson-Sköldberg et al. 2013) werden im Folgenden noch kurz einige besondere Aspekte in der Forschung zum Design-Prozess angesprochen, die Unternehmen in der eben skizzierten Situation helfen und auch schon als Bestandteile von Design-Prinzipien diskutiert aber selten als Forschungsgegenstand expliziert werden.

Als Designerly Thinking wird beschrieben, dass sich Denken und Vorgehensweise eines Designers und eines Ingenieurs zum Teil grundsätzlich unterscheiden. Designerly Thinking ist Kernaspekt des immer populäreren Design Thinking (Brown 2008), das Prinzipien des Designs (z. B. aus der Produktgestaltung) auf viele Problemstellungen von Unternehmen überträgt.

Viele DSR-Ansätze in der Wirtschaftsinformatik nutzen schon heute bewusst oder unbewusst Ansätze des Designerly Thinking oder Design Thinking (Redlich 2020).

So entspricht das ,sinnhafte (meaningful)“ (Krippendorff 2006) Artefakt, das aus dem Designerly Thinking entstehen soll, dem des Action Design Research: Ensemble-Artefakte, die Dienstleistungen, IT-Artefakte, Prozesse, Menschen und Organisationen umfassen. Auch berücksichtigen, wie im Folgenden beschrieben, Design-Prozesse von Informationssystemen neben IS-spezifischen Ansätzen bereits heute häufig solche, die auf die Vorgehensweise eines Designers zurückzuführen sind.

Die so genannten Wicked Problems (Rittel und Webber 1973), die das Designerly Thinking als Problemstellung thematisiert, werden schon in den Grundzügen des 
Design Science Research angesprochen - aber wenig im Prozess der Gestaltung adressiert. Wicked Problems sind Probleme, wie sie in der heutigen volatilen, unsicheren und komplexen Unternehmensumwelt immer häufiger auftreten. Sie können kaum in kleinere Probleme strukturiert, genau definiert oder langfristig sicher gefasst werden. Damit gibt es keine einzige beste Lösung für Wicked Problems, sondern die sich verändernde Umwelt sowie immer neues Wissen erfordern aufgrund der Wichtigkeit des Problems, dass Menschen in gemeinsamen Entscheidungsprozessen rasch zu immer neuen, besseren Lösungen gelangen müssen, die zwar immer auf der Kenntnis verschiedener Lösungsmöglichkeiten basieren, aber trotzdem kein langfristiges Lernen aus Fehlern zulassen.

Designerly Thinking und Design Thinking schlagen neben der im Action Design Research geforderten Beteiligung von Stakeholdern besondere Design-Prozesse für Wicked Problems sowie die gemeinsame Arbeit an einem Design (Thinking) Mindset (Dosi et al. 2018) vor. Letzteres akzeptiert Ambiguitäten und strebt trotzdem risikobereit sowie im Vertrauen auf kreative Ansätze nach raschen Lösungen. Dabei steht immer der Mensch als Ziel der Problemlösung im Mittelpunkt, was dazu führt, dass gegenseitige Empathie auch unterschiedliche Ansichten oder Fehler überwiegt.

Der Design-Prozess übernimmt in der Problemidentifikation, neben der Ziel- und Aufgabenformulierung, die Prinzipien des wiederholten Framing und Reframing aus dem Design. Zur Beteiligung von Stakeholdern tritt die Arbeit an einem gemeinsamen Shared Mental Model, das die gedankliche Einbettung des Problems sowie von Lösungsmöglichkeiten bei allen Partnern in Einklang bringt. Zur Induktion und Deduktion in der Lösungsfindung tritt die Abduktion - als Ansatz, Lösungsansätze aus anderen Bereichen zu erwägen, sowie das abwechselnde kreative Divergieren und strukturierte Konvergieren (Redlich 2020).

Heutige Unternehmen sehen sich mit Wicked Problems konfrontiert. Sie verfolgen Zielstellungen, die weit über Effizienzkriterien hinausgehen und stehen verschiedensten, häufig gestärkten Stakeholdern und einem höchst komplexen und unsicheren Umfeld gegenüber. Design-Prozesse, die potenziell auch Ansätze des Designerly Thinking berücksichtigen, sind damit sowohl ein wesentlicher Bereich der Forschung in der Wirtschaftsinformatik als auch eine Chance für die Praxis.

Mit dem Design Science Research und dem Action Design Research, die potenziell in Design-Theorien münden und zunehmend auch komplexe Design-Prozesse im Unternehmen unterstützen, hat die Wirtschaftsinformatik damit heute das Potenzial, Forschung mit den Erfordernissen der Praxis in Einklang zu bringen.

\section{Kollaborationsformen zur Forschung mit der Praxis}

Nach der bislang eher forschungsmethodischen Betrachtung stehen im Folgenden die Organisationsformen der Zusammenarbeit von Hochschulen und anderen Forschungseinrichtungen (im Folgenden verkürzt als Hochschulen bezeichnet) mit Praxispartnern aus Wirtschaft und Verwaltung (im Folgenden verkürzt als Unternehmen bezeichnet) im Vordergrund. Dabei werden die Ergebnisse einer Studie von Schubert et al. (2015) vorgestellt, die auf Basis einer Umfrage und einer anschließenden qualitativen Validierung durch Forscher verschiedene „Archetypen“ der Kollabora- 
tion von Hochschulen mit Unternehmen (siehe Tab. 2) identifiziert und in einem Framework (siehe Abb. 2) verdichtet haben.

Die „Blue Ocean“-Forschung, benannt nach dem Bestseller von Kim und Mauborgne (2015), zielt auf radikale Innovationen und das Eindringen in unbekannte Märkte mit völlig neuen Produkten oder Diensten. Blue-Ocean-Forschung kommt insbesondere für große erfolgreiche Unternehmen in Frage, die sich auf langfristige Vorhaben einlassen können, welche kurzfristig keine unmittelbar umsetzbaren Ergebnisse liefern. Forscherpersönlichkeiten, die in dieser Art von Kollaboration tätig werden, sind typischerweise selbst eher extrovertiert, umgeben von einer großen und stabilen Gruppe an Forschern (Post-Docs und Doktoranden) und erfahren genug, um in einem industriellen Kontext mit überzeugenden Ergebnissen aufwarten zu können. Die Motivation dieser Forscher entspringt dem potenziellen Impact ihrer Forschung und dem interessanten und herausfordernden Forschungsproblem. Zugang zu Daten bzw. empirischen Evaluationsmöglichkeiten und auch die Relevanz der Forschung können auch von Bedeutung sein. In methodischer Hinsicht sind hier häufig die induktive Fallstudien- und Aktionsforschung, aber auch DSR anzutreffen (Schubert et al. 2015).

Die Blue-Ocean-Forschung findet sich, ebenso wie die folgenden drei Typen, in bilateralen Szenarien, in denen eine Hochschule mit einer Einrichtung aus der Praxis zusammenarbeitet (Abb. 2, links oben).

In der Variante Beratung übernimmt die Forschungseinrichtung in vielen Aspekten die Rolle eines klassischen Beraters (siehe Abschn. 2.2), wobei für sie auch ein über den ökonomischen Nutzen hinausgehender Wert aus dem Projekt entsteht. Die typischerweise privatwirtschaftliche Finanzierung kommt je nach rechtlicher Ausgestaltung der Hochschuleinrichtung zugute oder den durchführenden Forschern persönlich im Rahmen einer Nebentätigkeit. An Hochschulen für angewandte Wissenschaften kann dies in Praxissemestern geschehen und dient dort auch der Erhaltung der „Praxisfähigkeit“ der für ihre in der Praxis erworbene Expertise berufenen Professoren. Forscherpersönlichkeiten, die dieser Art der Kollaboration besonders gerne nachgehen, gründen häufig auch ihre eigenen Beratungsunternehmen. Trotz möglicher Kritik an diesen Ansätzen ist ein Nutzen für die Forschungseinrichtung im Erkennen von relevanten Forschungsfragen, in der Absicherung ihrer Relevanz, verbesserten Forschungsmöglichkeiten für Doktoranden und in einem erheblichen Rückfluss an interessantem Material zurück in die Hochschule zu sehen, das insbesondere in der Lehre befruchtend eingesetzt werden kann (Schubert et al. 2015).

Bei der Form Entwicklung geht es im Ergebnis der Forschung um IT-Artefakte, oftmals Softwaresysteme, die zu Produkten des beteiligten Unternehmens werden sollen oder in seine vorhandenen Produkte eingehen. Das Unternehmen finanziert typischerweise die Entwicklungsarbeit auf Seiten der Hochschule und hat kein Interesse daran, dass die Ergebnisse der Allgemeinheit oder der Wissenschaftscommunity zur Verfügung gestellt werden. In Kollaborationen dieses Formats spielt daher die Regelung von Verwertungsrechten eine große Rolle. Methodisch handelt es sich konsequenterweise um DSR-Projekte, die, auch wenn finale Produkte daraus resultieren, dennoch Publikationsmöglichkeiten bieten (Schubert et al. 2015).

Die Langzeitpartnerschaft basiert auf einem vertrauensvollen Miteinander über einen längeren Zeithorizont hinweg, in dem die Partner, wenn sich die Gelegen- 


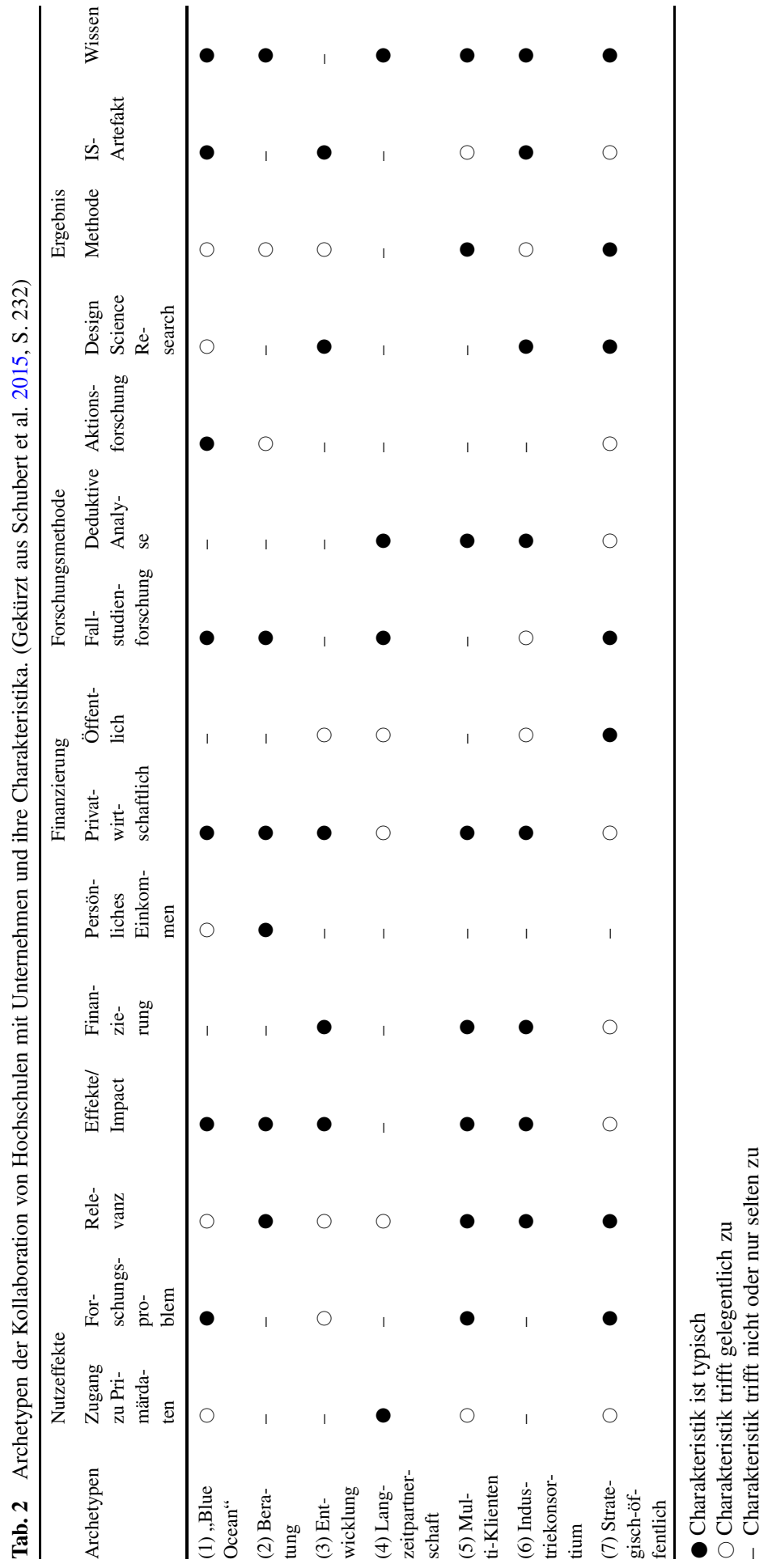


Abb. 2 Archetypen-Framework. (Schubert et al. 2015, S. 236)
1

Anzahl Unternehmen

\begin{tabular}{|c|c|}
\hline $\begin{array}{l}\text { (1) Blue Ocean } \\
\text { (2) Beratung } \\
\text { (3) Entwicklung } \\
\text { (4) Langzeitpartnerschaft }\end{array}$ & (5) Multi-Klienten \\
\hline (6) Industriekonsortium & (7) Strategisch-öffentlich \\
\hline
\end{tabular}

heit ergibt, mit Ideen aufeinander zukommen und Projekte initiieren, um willen des wissenschaftlichen Ergebnisses, typischerweise nicht, um in erster Linie Mittel zu akquirieren. Forscher, die in diesem klassischen Format arbeiten, sind überzeugt davon, dass Forschung in ihrem Fach aus der Praxis heraus motiviert sein sollte und der Praxiskontext hilfreich für die Theorieentwicklung ist. Fallstudienforschung (in induktiver oder deduktiver Form) ist in diesen Kontexten eine häufig eingesetzte Forschungsmethode. Bei der Langzeitpartnerschaft kann die Finanzierung unterschiedlicher Natur sein, aber u. a. auch darauf fußen, dass Forscher aus ihrem forscherischen Interesse und der wissenschaftlichen Nützlichkeit heraus ihre Arbeitszeit einbringen. Forscher in diesem sehr klassischen Format suchen in der Regel die bilaterale Beziehung und sind zurückhaltend beim Aufbau größerer Konsortien, weil sie darin eine Gefährdung ihres frei bestimmten und interessensgetriebenen Engagements sehen (Schubert et al. 2015).

In der Variante Multi-Klienten wird die Bilateralität zugunsten mehrerer Praxispartner aufgegeben, so dass ein universitätsseitig geriebenes Konsortium entsteht. Typischerweise haben die Praxispartner komplementäre Interessen in einem Themenfeld oder es handelt sich um vorwettbewerbliche Themen. Die Kollaboration der Praxispartner kann intensiv sein, ist aber auch in loseren Formen anzutreffen. Die Finanzierung erfolgt in der Regel durch einen Beitrag, den jeder der Praxispartner zahlen muss. Forschungsergebnisse sind typischerweise nicht exklusiv, werden aber den Partnern früher und möglicherweise in anderen Formaten zu Verfügung gestellt (Schubert et al. 2015).

Das Industriekonsortium beschreibt eine Form, in der ein marktführendes, großes Unternehmen eine radikale Innovation plant, hierzu aber nicht über die passende Expertise verfügt und auch kaum Chancen hat, diese Expertise in Form von Mitarbeitern zu gewinnen. Ein Weg trotzdem voranzukommen, kann darin bestehen, Wissensträger aus Forschungseinrichtungen in einem Konsortium zusammenzuführen. Trotz des Risikos solcher Vorhaben rechtfertigen die Chancen dieses Ansatzes verbunden mit dem immensen Impact diese Vorgehensweise. Typischerweise werden solche Konsortien vom Unternehmen selbst finanziert, aber auch öffentliche Finanzierung ist denkbar, z. B. wenn das Thema in ein von Seiten der Politik als strategisch erachtetes Feld passt (Schubert et al. 2015).

Genau dies steht bei der strategisch-öffentlichen Forschung im Vordergrund. Themenfelder, die auf Bundes- oder EU-Ebene als strategisch erachtet werden, werden in großen Förderprogrammen abgebildet (z. B. die EU-Framework-Programme oder auch diverse Programme des BMBF), auf die sich größere Konsortien bestehend aus mehreren Forschungs- und Praxispartnern bewerben (typischerweise in einem n:m- 
Setup). Praxispartner können in diesen Gruppen aktiv Forschung betreiben oder auch als „Verwertungspartner“ fungieren, denn meistens wird von öffentlicher Seite die Stärkung der Wettbewerbsfähigkeit von Unternehmen/Branchen/Ländern verfolgt. Die gebildeten Konsortien können so groß sein, dass sie sich für die jeweiligen Arbeitspakete in kleinere Gruppen aufteilen. Auch die Interessenslagen und Motivationen der beteiligten Parteien müssen nicht immer kongruent sein. Forscher, die in diesen Formaten tätig sind, sind ausgesprochen gute Netzwerker und verstehen es, verschiedenste Parteien zusammenzubringen und das Miteinander, trotz möglicher Interessenskonflikte, voranzutreiben (Schubert et al. 2015).

Unter den vorgestellten sieben Archetypen handelt es sich bei den zuletzt genannten drei Formen jeweils um Konsortialforschung. Ein Beitrag, der diese Organisationsform nicht, wie hier bei den Archetypen geschehen, nach dem treibenden Partner differenziert, sondern in methodischer Hinsicht für den Fall gestaltungsorientierter Forschung zu einer Methode für die Zusammenarbeit von Forschung und Praxis ausgestaltet, findet sich in Österle und Otto (2010). Der Beitrag kombiniert also Fragen der Organisationsform mit solchen der Forschungsmethodik. Er ist damit ein typisches Beispiel für Beiträge, die den Forschern und Praktikern in der Wirtschaftsinformatik aufzeigen, wie sie gemeinsam praxisrelevante Forschung durchführen können.

\section{Weitere Wege zur Praxisrelevanz}

Auch wenn in diesem Beitrag methodische und organisatorische Ansätze hervorgehoben wurden, die Forschung gemeinsam mit der und für die Praxis besonders unterstützen, ist es keineswegs unsere Absicht, nicht behandelten Ansätzen und den entsprechend hervorgebrachten Ergebnissen Praxisrelevanz abzusprechen. Praxisrelevanz kann natürlich auch in ganz anderen Forschungsformaten herbeigeführt werden und ist in der Wirtschaftsinformatik als angewandter Disziplin immer von besonderer Bedeutung. Deshalb beschäftigen sich Forscher z. B. gezielt auch damit, wie Praxisrelevanz explizit gesteigert werden kann. Im Folgenden behandeln wir Beispiele zu drei prinzipiellen Möglichkeiten:

- Ergänzungen zu Forschungsmethoden,

- Steigerung der Forschungs,,konsumierbarkeit“,

- Verlassen klassischer Forschungspfade.

Dabei subsumieren wir unter Praxisrelevanz (in Anlehnung an Rosemann und Vessey (2008), die sich wiederum auf Arbeiten von Klein et al. (2006) sowie Benbasat und Zmud (1999) stützen) folgende drei Dimensionen:

- Wichtigkeit (importance) im Sinne der zeitgerechten Adressierung eines Problems aus der Praxis,

- Zugänglichkeit (accessibility) im Sinne eines Fokus auf verständlich beschriebene Forschungsergebnisse (im Gegensatz zu einem Fokus auf Forschungsmethoden) sowie 
- Eignung (suitability) im Sinne von Lösungsansätzen, die das Potenzial haben, auf ein Problem in einem konkreten Praxiskontext angewendet oder perspektivisch für eine Innovation genutzt zu werden.

Wichtigkeit und Eignung adressieren den Inhalt der Forschung, wobei Wichtigkeit als Voraussetzung von Eignung betrachtet werden kann. Forschung, deren Inhalt von Praktikern nicht als wichtig erachtet wird, wird auch kaum als geeignet für die Lösung ihrer Probleme betrachtet werden können. Die Zugänglichkeit hingegen adressiert die Frage, wie Forschungsergebnisse kommuniziert werden (Rosemann und Vessey 2008).

Neben diesen drei Kriterien sollten bei praxisrelevanter Forschung auch Fragestellungen der Nachhaltigkeit, im Sinne von Verantwortung und Ethik, nicht außer Acht gelassen werden. Wir betrachten diese Dimension jedoch weniger als konstituierend, sondern eher als aus der Praxisrelevanz folgend.

\subsection{Ergänzungen zu Forschungsmethoden}

Rosemann und Vessey (2008) adressieren in erster Linie die Wichtigkeit von Forschung für Praktiker und stellen so genannte Applicability Checks vor, die einen klassischen Forschungsprozess ergänzen oder erweitern können, ohne die eingesetzten Forschungsmethoden darauf anpassen zu müssen. Sie sind geeignet für viele Formen empirischer Forschung, bei Aktionsforschung jedoch eher überflüssig.

In einem klassischen Forschungszyklus sehen sie zwei mögliche Zeitpunkte vor, in dem diese Anwendbarkeitsprüfungen vorgenommen werden können: bei ,junger" Forschung am Ende eines Zyklus, wenn Forschungsergebnisse vorliegen, diese aber noch nicht publiziert sind, oder bei ,reifer“ Forschung, zu der bereits viele Publikationen entstanden sind, vor Einstieg in einen neuen Forschungszyklus, um sicherzustellen, dass in ,relevanter“ Richtung weitergearbeitet wird. Applicability Checks selbst werden von Rosemann und Vessey (2008) als Methode beschrieben, die auf einem sieben-schrittigen Ablaufplan basiert, und Fokusgruppen mit Methoden zur Ideenfindung kombiniert. Die Methode der Applicability Checks wird von Rosemann und Vessey an reifer Forschung illustriert, nämlich dem DeLone/Mc Lean IS Success Model (DeLone und McLean 2003) und ist leicht auf eigene Projekte und Forschungsergebnisse übertragbar.

Eine weitere Form der Ergänzung von Forschungsmethoden mit der Zielsetzung, Eignung zu erhöhen, kann durch empirische Bausteine, die moderne Technologien nutzen, erfolgen. So kann die Eignung von Lösungen im Sinne beispielsweise ihrer Akzeptanz oder der Anwendbarkeit durch moderne Informationstechnik getestet werden, die in neuer Weise die Schnittstelle zwischen Mensch und Technik adressiert. So ermöglichen beispielsweise Systeme der Virtual oder Augmented Reality dem Nutzer eine Immersion in zukünftige Szenarien, die so noch nicht existieren. Oder ausgereifte Sensorik kann, auch außerhalb einer Laborumgebung, die Reaktionen von Nutzern neuer Informationssysteme erfassen. Hier trägt innovative Informationstechnologie in Nutzerstudien also selbst dazu bei, Eignung für die Praxis im Forschungsprozess anders und ggf. früher festzustellen, als dies bislang möglich 
war. Solche Bausteine könnten zukünftig vermehrt in Forschungsprozesse integriert werden.

\subsection{Steigerung der Forschungskonsumierbarkeit}

Hier geht es in erster Linie um die Dimension Zugänglichkeit von Forschung für Praktiker, einer Zielsetzung, der sich die Zeitschrift „HMD - Praxis der Wirtschaftsinformatik“ per se verpflichtet fühlt. Schon Autoren wie Robey und Markus (1998) sowie Benbasat und Zmud (1999) haben sich mit der Frage, wie Forschungsergebnisse praxisgerecht präsentiert werden können und welche Kanäle/Medien Forscher bedienen sollten, beschäftigt. Sie geben eine Fülle von nützlichen Hinweisen, die nach wie vor Gültigkeit haben und die nahezu jeder Forscher als Verfasser und Reviewer von Forschung oder als Herausgeber von Journalen berücksichtigen sollte. Neben Schreibstil (Sprache, Illustrationen, Storyline etc.) und Fokus (auf Output der Forschung, nicht Input) wird auch das Problem unterschiedlicher Terminologie behandelt, weil sich Fachbegriffe in Forscher- und Praktikercommunities oftmals unterscheiden (z.B. IS vs. IT, Backsourcing vs. Insourcing) und somit ein thematischer Fit erst gar nicht oder nur über Umwege herbeigeführt werden kann. Wie ein solcher Themen- und Terminologieabgleich erfolgen kann, zeigen methodische und exemplarische Arbeiten wie von Marrone und Hammerle (2016, 2017), Brandt et al. (2018) sowie von Bary et al. (2018).

Insbesondere im Bereich der qualitativen Forschung gibt es eine Vielzahl von Arbeiten, die adressieren, wie Forschungsergebnisse „gut“ (im Sinne von anschaulich, mutig, kreativ ...) präsentiert werden können (z. B. Mitchell und Clark 2018), angefangen von Kurzbeiträgen bis hin zu kompletten Büchern (siehe z. B. die Empfehlungen in (Pacheco-Vega 2018)).

\subsection{Verlassen klassischer Forschungspfade}

Grover und Lyytinen (2015) zeigen in ihrem Beitrag auf, wie die dominante Form der Wissensproduktion in der Disziplin IS noch immer abläuft: Theorien aus Referenzdisziplinen werden herangezogen und leicht kontextualisiert (wie im Beispiel der eingangs erwähnten Forschung mit dem Technology-Acceptance-Modell), d.h. dass das IS-spezifische sich lediglich in einer abhängigen oder einer Mediator- bzw. Moderatorvariablen manifestiert und dabei weitgehend exogen bleibt. Dabei entstehen in großer Zahl leicht angepasste Midrange-Theorien, die weder als spezifische IS-Theorien bezeichnet werden können, noch sich gut konsolidieren lassen und oftmals wenig originelle Ergebnisse hervorbringen. Grover und Lyytinen geben eine gute Darstellung, warum diese Entwicklung trotz wiederholter vorheriger Aufrufe nicht leicht zu stoppen ist. Sie schlagen vor, diese ewig und immer wieder praktizierte Routine in zwei extreme Richtungen hin zu durchbrechen. Davon geht eine in Richtung ,mutigerer“ Theorieentwicklung und eine andere in Richtung ,datengetriebener" Forschung. Während ersteres durch originellere und disziplinspezifische Theorien mehr Nutzen für die Forschung verspricht, geht es bei letzterem um die Nutzung und Nutzen in der Praxis. Die Autoren plädieren für größere Offenheit bzgl. rein datengetriebener induktiver Forschung, die interessante und neue Phänomene 
hervorbringt, mit Praxisevidenz arbeitet, aber nicht immer mit wissenschaftlicher Literatur fundiert werden kann oder gar schon auf theoretische Erklärungszusammenhänge rekurriert:

As a field in which dynamics of IT innovation and use catalysts constantly change in human enterprise, we should be keen and acute observers of practice independent of prior theoretical baggage. Pure observation, description, and identification of patterns are valid modes of research ... (Grover und Lyytinen 2015, S. 287)

Die Autoren identifizieren insgesamt drei Ausgangspunkte von rein-empirischen Studien: die Welt wie sie ist (,as-is“), wie sie sein wird (,will-be“) und die Welt, die neugierig macht (,curious world“) (Grover und Lyytinen 2015). Neben anschaulichen und reichhaltigen Beschreibungen von IT-Phänomen verspricht gerade die zweite Form neben Wichtigkeit auch das Kriterium Eignung für die Praxis zu adressieren. Durch die Darstellung einzigartiger Nutzung von IT können Hinweise darauf gegeben werden, wie perspektivische Lösungen aussehen können, auch wenn sie noch in weiter Ferne liegen. Gerade dieser Blick nach vorne ist einer, den Praktiker von Forschung erwarten und hören wollen. Es ist nicht immer die Umsetzung sofort, sondern das Absehenkönnen, was langfristig möglich sein wird, was Praktiker inspiriert, sich mit Forschung auseinanderzusetzen. Die neugierige Welt, die widersprüchliche, kontraintuitive Theorien und sich widersprechende Ergebnisse hervorbringt, ohne dass wir verstehen warum, ist ebenfalls eine, die langfristig Ergebnisse hervorbringen kann, die weitaus revolutionärer sind, als wir dies in unseren angestammten Pfaden gewöhnt sind. Mit diesem Potenzial zur Innovation ist möglicherweise auch größere Eignung für die Praxis gegeben.

Die in diesem Abschnitt vorgestellten exemplarischen Arbeiten und Aufsätze zeigen in beeindruckender Weise, dass auch Forscher, die dominant theoriebasiertkonfirmatorisch arbeiten, ein starkes Bewusstsein für Praxisrelevanz aufweisen können und dem notwendigen Streben danach eine große Bedeutung beimessen. So hoffen wir aufgezeigt zu haben, dass Fragen wie „Couldn't we just ignore the practitioners and hope they go away?", die Robey und Markus (1998, S. 8) noch als allgegenwärtig charakterisieren, hoffentlich bald der Vergangenheit angehören.

\section{Fazit}

Der Beitrag postuliert, dass praxisorientierte Forschung in der Wirtschaftsinformatik auf verschiedenen Wegen entstehen kann. Für den Forschenden zeigt er eine Reihe von Forschungsmethoden auf, die sich besonders gut eignen, um für die Praxis und sogar gemeinsam mit der Praxis zu forschen und dabei die eigene Forschung sowohl in der Wissenschaftscommunity zu platzieren als auch für Unternehmen wertvoll zu machen. Letzteres erfordert aber noch eine Reihe von Rahmenbedingungen, die in Forschungsprojekten mit der Praxis beachtet und organisiert werden müssen. Am Beispiel verschiedener Archetypen von Kollaborationsformen haben wir gängige Muster hierfür vorgestellt. Sie zeigen vor allem dem Praktiker auf, welche Konstellationen es ihm, aber auch dem Forschenden, erlauben, mit gemeinsamen 
Projekten die eigenen Ziele zu verfolgen. Grundsätzlich sind dies aber nicht die einzigen Wege, wie Praxisrelevanz in der Forschung sichergestellt werden kann. Neben Erweiterungsansätzen, die sich mit verschiedensten Forschungsmethoden verknüpfen lassen, dem Arbeiten an der Konsumierbarkeit der Forschungsergebnisse und dem Verlassen tradierter Forschungspfade haben wir nur eine kleine Auswahl von Mechanismen vorgestellt, an denen es neben Methoden und Kollaborationsformen zu arbeiten gilt. Diese Diskussion fortzuführen, die Entwicklung solcher Ansätze ebenso wie gemeinsame Forschungsprozesse anzuregen und die dabei gemachten Erfahrungen auszutauschen, wünschen wir uns von Seiten der Forschung, aber auch der Praxis, am besten von beiden zusammen.

Funding Open Access funding provided by Projekt DEAL.

Open Access Dieser Artikel wird unter der Creative Commons Namensnennung 4.0 International Lizenz veröffentlicht, welche die Nutzung, Vervielfältigung, Bearbeitung, Verbreitung und Wiedergabe in jeglichem Medium und Format erlaubt, sofern Sie den/die ursprünglichen Autor(en) und die Quelle ordnungsgemäß nennen, einen Link zur Creative Commons Lizenz beifügen und angeben, ob Änderungen vorgenommen wurden.

Die in diesem Artikel enthaltenen Bilder und sonstiges Drittmaterial unterliegen ebenfalls der genannten Creative Commons Lizenz, sofern sich aus der Abbildungslegende nichts anderes ergibt. Sofern das betreffende Material nicht unter der genannten Creative Commons Lizenz steht und die betreffende Handlung nicht nach gesetzlichen Vorschriften erlaubt ist, ist für die oben aufgeführten Weiterverwendungen des Materials die Einwilligung des jeweiligen Rechteinhabers einzuholen.

Weitere Details zur Lizenz entnehmen Sie bitte der Lizenzinformation auf http://creativecommons.org/ licenses/by/4.0/deed.de.

\section{Literatur}

von Bary B, Westner M, Strahringer S (2018) Do researchers investigate what practitioners deem relevant? Gaps between research and practice in the field of information systems backsourcing. In: 2018 IEEE 20th conference on business Informatics (CBI), S 40-49

Baskerville RL (1997) Distinguishing action research from participative case studies. J Syst Inf Technol 1:24-43. https://doi.org/10.1108/13287269780000733

Baskerville R, Myers MD (2004) Special issue on action research in information systems: making is research relevant to practice-foreword. Mis Q 28:329-335. https://doi.org/10.2307/25148642

Baskerville R, Lyytinen K, Sambamurthy V, Straub D (2011) A response to the design-oriented information systems research memorandum. Eur J Inf Syst 20:11-15. https://doi.org/10.1057/ejis.2010.56

Benbasat I, Zmud RW (1999) Empirical research in information systems: the practice of relevance. Mis Q 23:3-16. https://doi.org/10.2307/249403

Boyer EL (1996) The scholarship of engagement. Bull Am Acad Arts Sci 49:18-33. https://doi.org/10. $2307 / 3824459$

Brandt T, Ketter W, Kolbe LM et al (2018) Smart cities and digitized urban management. Bus Inf Syst Eng 60:193-195. https://doi.org/10.1007/s12599-018-0537-1

Brown T (2008) Design thinking. Harv Bus Rev 86:84-92

Chandra L, Seidel S, Gregor S (2015) Prescriptive knowledge in IS research: conceptualizing design principles in terms of materiality, action, and boundary conditions. In: 2015 48th Hawaii International Conference on System Sciences (HICSS), S 4039-4048

Darke P, Shanks G, Broadbent M (1998) Successfully completing case study research: combining rigour, relevance and pragmatism. Inf Syst J 8:273-289. https://doi.org/10.1046/j.1365-2575.1998.00040.x

DeLone WH, McLean ER (2003) The delone and mclean model of information systems success: a tenyear update. J Manag Inf Syst 19:9-30. https://doi.org/10.1080/07421222.2003.11045748

Dosi C, Rosati F, Vignoli M (2018) Measuring design thinking mindset, S 1991-2002 
Fischer C, Winter PDR, Wortmann DF (2010) Design theory. Bus Inf Syst Eng 2:387-390. https://doi.org/ 10.1007/s12599-010-0128-2

Gregor S (2002) Design theory in information systems. Australas J Inf Syst. https://doi.org/10.3127/ajis. v10i1.439

Gregor S, Hevner AR (2013) Positioning and presenting design science research for maximum impact. Mis Q 37:337-A6

Gregor S, Jones D (2007) The anatomy of a design theory. J Assoc Inf Syst 8:313-335

Grover V, Lyytinen K (2015) New state of play in information systems research: the push to the edges. Mis Q 39:271-296

Heier H, Strahringer S (2006) Knowledge management systems and organizational change management: the case of Siemens sharenet. In: Reimer U, Karagiannis D (Hrsg) Practical aspects of knowledge management. Springer, Berlin Heidelberg, S 97-105

Hevner AR (2007) A three cycle view of design science research. Scand J Inf Syst 19:87-92

Hevner A, vom Brocke J, Maedche A (2018) Roles of digital innovation in design science research. Bus Inf Syst Eng. https://doi.org/10.1007/s12599-018-0571-z

Hevner AR, March ST, Park J, Ram S (2004) Design science in information systems research. Mis Q 28:75-105. https://doi.org/10.2307/25148625

Iivari J (2015) Distinguishing and contrasting two strategies for design science research. Eur J Inf Syst 24:107-115. https://doi.org/10.1057/ejis.2013.35

Johansson-Sköldberg U, Woodilla J, Çetinkaya M (2013) Design thinking: past, present and possible futures. Creat Innov Manag 22:121-146. https://doi.org/10.1111/caim.12023

Kim WC, Mauborgne R (2015) Blue ocean strategy, expanded edition: how to create uncontested market space and make the competition irrelevant, expanded. Harvard Business Review Press, Boston

Klein G, Jiang J, Saunders C (2006) Leading the horse to water. Commun Assoc Inf Syst. https://doi.org/ 10.17705/1CAIS.01813

Kobus J, Westner M, Strahringer S, Strode D (2018) Enabling digitization by implementing Lean IT: lessons learned. TQM J 30:764-778. https://doi.org/10.1108/TQM-02-2018-0026

Krippendorff K (2006) The semantic turn: a new foundation for design. CRC Press, Boca Raton

Maedche A, Gregor S, Morana S, Feine J (2019) Conceptualization of the problem space in design science research. In: Tulu B, Djamasbi S, Leroy G (Hrsg) Extending the boundaries of design science theory and practice. Springer, Cham, S 18-31

Marrone M, Hammerle M (2016) An integrated literature review: establishing relevance for practitioners

Marrone M, Hammerle M (2017) Relevant research areas in IT service management: an examination of academic and practitioner literatures. Commun Assoc Inf Syst. https://doi.org/10.17705/1CAIS. 04123

Mathiassen L, Nielsen PA (2008) Engaged scholarship in IS research. Scand J Inf Syst 20:3-20

Mitchell KM, Clark AM (2018) Five steps to writing more engaging qualitative research. Int J Qual Methods 17:1609406918757613. https://doi.org/10.1177/1609406918757613

Mueller SK, Mendling J, Bernroider EWN (2019) The roles of social identity and dynamic salient group formations for ERP program management success in a postmerger context. Inf Syst J 29:609-640. https://doi.org/10.1111/isj.12223

Österle H, Otto B (2010) Konsortialforschung. WIRTSCHAFTSINFORMATIK 52:273-285. https://doi. org/10.1007/s11576-010-0238-y

Österle H, Becker J, Frank U et al (2010) Memorandum zur gestaltungsorientierten Wirtschaftsinformatik. Schmalenbachs Z Betriebswirtsch Forsch 62:664-672. https://doi.org/10.1007/BF03372838

Österle H, Becker J, Frank U et al (2011) Memorandum on design-oriented information systems research. Eur J Inf Syst 20:7-10. https://doi.org/10.1057/ejis.2010.55

Pacheco-Vega R (2018) Six writing books to improve your qualitative methods prose. Int J Qual Methods 17:1609406918782045. https://doi.org/10.1177/1609406918782045

Peffers K, Tuunanen T, Niehaves B (2018) Design science research genres: introduction to the special issue on exemplars and criteria for applicable design science research. Eur J Inf Syst 27:129-139. https:// doi.org/10.1080/0960085X.2018.1458066

Redlich BM (2020) Performing design thinking virtually-A socio-cognitive view on virtual design thinking. Jacobs University Bremen, Bremen

Rittel HWJ, Webber MM (1973) Dilemmas in a general theory of planning. Policy Sci 4:155-169. https:// doi.org/10.1007/BF01405730

Robey D, Markus ML (1998) Beyond rigor and relevance: producing consumable research about information systems. Inf Resour Manag J 11:7-16. https://doi.org/10.4018/irmj.1998010101 
Rosemann M, Vessey I (2008) Toward improving the relevance of information systems research to practice: the role of applicability checks. Mis Q 32:1-22. https://doi.org/10.2307/25148826

Sarker S, Sarker S, Sahaym A, Bjørn-Andersen N (2012) Exploring value cocreation in relationships between an ERP vendor and its partners: a revelatory case study. Mis Q 36:317-338. https://doi.org/10. 2307/41410419

Sarker S, Xiao X, Beaulieu T, Lee AS (2018a) Learning from first-generation qualitative approaches in the IS discipline: an evolutionary view and some implications for authors and evaluators (PART 1/2). J Assoc Inf Syst 19:752-774. https://doi.org/10.17705/1jais.00508

Sarker S, Xiao X, Beaulieu T, Lee AS (2018b) Learning from first-generation qualitative approaches in the IS discipline: an evolutionary view and some implications for authors and evaluators (PART 2/2). J Assoc Inf Syst 19:909-923. https://doi.org/10.17705/1jais.00512

Schubert P, Andersen NB, Kilian T (2015) Archetypes for engaged scholarship in information systems. Int J Inf Syst Manag 1:219-239. https://doi.org/10.1504/IJISAM.2015.072296

Sein MK, Henfridsson O, Purao S et al (2011) Action design research. Mis Q 35:37-56. https://doi.org/10. 2307/23043488

Simon HA (1969) The sciences of the artfificial. MIT Press, Cambridge

Simon HA (1996) The sciences of the artificial, 3. Aufl. MIT Press, Cambridge

Van de Ven AH (2007) Engaged scholarship: a guide for organizational and social research. Oxford University Press, Oxford, New York

Walls JG, Widmeyer GR, El Sawy OA (1992) Building an information system design theory for vigilant EIS. Inf Syst Res 3:36-59

Winter R (2008) Design science research in Europe. Eur J Inf Syst 17:470-475. https://doi.org/10.1057/ ejis. 2008.44

Yin RK (2018) Case study research and applications, 6. Aufl. SAGE, Los Angeles 